\title{
Continuous Publication
}

Paulo Emílio V. de Miranda ${ }^{1}$

\author{
${ }^{1}$ Editor-in-Chief \\ Matéria Journal \\ E-mail: pmiranda@labh2.coppe.ufrj.br
}

For several years the journal Materia has subdivided its annual publication in four quarterly numbers or fascicles that take place every March, June, September and December each year. However, the flux of papers to the journal has grown very much; the number of papers published in 2016 was 3.5 times greater than that published five years ago. Because of that, some papers were submitted, reviewed and, upon being accepted, had to wait until the next publication date for one of the annual journal's numbers. But the present dynamics does not accept to wait for the publication of a paper. It has to be done the sooner the possible, once accomplished all procedures concerning peer reviewing, formatting and editing. Fortunately, SciELO has implemented a new publication procedure for its journals. It is the continuous publication, an innovative paper publishing possibility that does not wait the complete preparation of a fascicle or of any serial edition for publishing a paper, which contributes to speed up the publication process. The main objective of the continuous publication of papers is to accelerate communication of research work, thus contributing to make it available faster for reading and citation. A modification that comes with the continuous publication of papers is that page numbering is no longer utilized; such as it occurred with conventional printed publications and even with the digital ones that appeared in fascicles. All papers loose their page information in a continuous publication and receive an electronic location identifier, elocation-id, which is a unique identifier of the article in a fascicle. This element acts in a similar way to a page identifier to locate the document that does not possess a traditional page numbering.

From January 2017 on, matching the beginning of its volume 22, the journal Materia has adopted the continuous publication of papers. There will be no more waiting to publish a paper that has been accepted and edited. For example, Number 1 of Volume 22 begins publishing papers available in January and publishes them continuously until March, when that number is closed. Therefore, every paper accepted and edited between January and March is promptly published. From April on, a new sequence of continuous publication begins, which is closed in June. Then, the publication of the four annual numbers of the journal proceeds, aggregating papers continuously until a fascicle is closed to give initiation to the next one. The journal follows on utilizing the Digital Object Identifier, DOI, which is a number that identifies uniquely a digital document and guarantees its localization in the virtual network. The first paper published by the journal Materia with the new continuous publication procedure in January 2017 is entitled "Resonance method for predicting the mechanical properties of heat-treated Eucalyptus urophylla and Pinus oocarpa wood", with elocation-id e11772 and DOI 10.1590/S1517-707620170001.0104. 